\title{
A Rapid and Precise Method to Determine Cysteine Content in Food Materials using
}

\section{4-(aminosulfonyl)-7-fluoro-2,1,3-benzoxadiazole}

\author{
Hiroaki NishIUCHI ${ }^{*}$, Masanori KoHMURA and Hidehiko WAKABAYASHI \\ Ajinomoto Co., Inc., 1-1 Suzuki-cho, Kawasaki-Ku, Kawasaki-shi, Kanagawa 210-8681, Japan
}

Received October 30, 2010; Accepted April 20, 2011

A method to determine cysteine content in food materials using 4-(aminosulfonyl)-7-fluoro-2,1,3-benzoxidiazole (ABD-F) was compared to a method using $\mathrm{N}$-acridinyl maleimide (NAM). Cysteine in samples was derivatized with two fluorogenic reagents and analyzed using HPLC. The cysteine derivative with ABD-F gave a single peak while the cysteine derivative with NAM produced three peaks in standard. The cysteine content in model samples determined with ABD-F was nearly identical to the content determined with NAM. However, when the cysteine content of six types of food materials was measured, four samples were identical in each method, but two samples produced different results because the separation of cysteine derivatives with NAM was influenced by other compounds in the food samples. Since the ABD-F method gave the better separation, it can be useful for the determination of cysteine content in food materials.

Keywords: 4-(aminosulfonyl)-7-fluoro-2,1,3-benzoxidiazole (ABD-F), N-acridinylmaleimide (NAM), cysteine

\section{Introduction}

L-Cysteine, one of the 20 natural amino acids, is an important sulfur-containing compound because it is the only source of sulfur for cell metabolism in most organisms. Cysteine is indispensable for protein folding, protein assembly, and protein stability through disulfide bond formation with the reactive thiol residue. In addition to this metabolic importance, cysteine also plays an important industrial role. Cysteine has been used for industrial applications such as pharmaceuticals, cosmetics, bakery, and flavorings, resulting in consumption of approximately 4000 tons/y globally (Wada et al., 2006). For example, cysteine was reported to be effective as a reducing agent in production of French bread, crackers, and cookies (Grandvoinnet et al., 1979). In the beverage industry, cysteine was effective in preventing browning of fruit juice, such as grape juice or pear juice, during concentration (Skalski and Sistrunk, 1974; Montgomery, 1983.) In addition, cysteine prevented the development of off-flavor in stored orange juice (Naim et al., 1993). In flavor chemistry, cysteine is an important source of sulfur in a variety of aromas (Strakenmann et al., 2008). Thus, cysteine will continue

*To whom correspondence should be addressed. E-mail: hiroaki_nishiuchi@ajinomoto.com to play an important role in the food industry. Therefore, a reliable method to determine the cysteine content in food materials is necessary.

Several methods have been developed to determine cysteine content. Previously, mercaptide-forming reactions using metal ions were used. However, more recent methods use fluorescent reagents to react with thiol residues. For example, NAM, a maleimide reagent, provides blue fluorescence by reaction with thiol compounds and has been used for analysis of thiol compounds such as cysteine or glutathione (Nara et al., 1978; Takahashi et al., 1979; Meguro et al., 1996). In addition, NAM has been used to determine cysteine content in commercial yeast extracts (Kuroda et al., 1997). In contrast, ABD-F, a reagent with a halogenobenzofurazan structure, has been used to determine cysteine content (Toyo'oka et al., 1984; Reddy et al., 1993), but cysteine labeling by ABD-F was reported to be unstable under certain conditions (Treuheit et al., 1993). Although these two fluorescent reagents are often used, their performance has not been compared.

This report compares the ABD-F and NAM methods to determine the cysteine content in food materials.

\section{Material and Methods}

Chemicals Reagent grade ABD-F, NAM, L-cyste- 
ine, acetate, acetone, sodium borate, ethylenediamine -N,N,N', N'-tetraacetic acid disodium salt dehydrate $($ EDTA $\cdot \mathrm{Na})$, and trifluoroacetic acid (TFA) were purchased from Wako Pure Chemical Industries (Tokyo, Japan). HPLC grade methanol and acetonitrile were purchased from Wako Pure Chemical Industries. Powder forms of food materials were obtained from several manufacturers.

Preparation of model samples Model samples in liquid form were prepared by dissolving reagent grade yeast extract from Becton, Dickinson, and Company (Franklin Lakes, New Jersey, USA) at the predetermined concentration and then adding cysteine solution. A model sample in powder form was prepared by mixing cysteine with the yeast extract. The yeast extract and cysteine were dissolved to prepare an analytical sample and then the concentration was adjusted to prevent loss of powder.

HPLC The high performance liquid chromatograph consisted of a liquid chromatograph pump LC-20AD, degasser DGU-20A 5 , auto sampler SIL-20AC $\mathrm{HT}_{\mathrm{HT}}$, column oven CTO-20A, fluorescence detector RF-10A $\mathrm{A}_{\mathrm{XL}}$, communications bus module CBM-20A, and work station LCsolution (Shimadzu Corp., Kyoto, Japan). An isocratic elution system was employed. Operation of the HPLC unit, as well as data collection and calculations were performed by the work station.

Determination of cysteine by HPLC using $A B D-F$

HPLC conditions and ABD-F derivative conditions were determined by referring to previous reports (Toyo'oka et al., 1984; Reddy et al., 1997) and the reagent manufacturer's instructions. The procedure involved: samples were dissolved in $5 \mathrm{mmol} / \mathrm{L}$ sodium acetate buffer containing $2 \mathrm{mmol} / \mathrm{L}$ EDTA ( $\mathrm{pH} 4.0)$ at predetermined concentrations. ABD-F was dissolved in acetonitrile and added into $0.1 \mathrm{~mol} / \mathrm{L}$ sodium borate buffer containing $2 \mathrm{mmol} / \mathrm{L}$ EDTA ( $\mathrm{pH} 8.0$ ) so as to prepare $0.5 \mathrm{mmol} / \mathrm{L}$ ABD-F solution. Then the cysteine standard solution or the sample solution was mixed with three volumes of ABD-F solution (final $\mathrm{pH}$ was in the range of 7.9 to 8.0 ) and incubated for $15 \mathrm{~min}$ at $50^{\circ} \mathrm{C}$. Then the reactant was filtered through a $0.45 \mu \mathrm{m}$ filter and $10 \mu \mathrm{L}$ of the filtered reactant was injected into the mobile phase consisting $17 \%$ acetonitrile and $0.1 \%$ TFA. The flow rate of the mobile phase was $1.4 \mathrm{~mL} / \mathrm{min}$, and the separation was performed using an Inertsil ODS-3 column [3 $\mu \mathrm{m} 4.0 \mathrm{~mm} \phi \times 100 \mathrm{~mm}$; GL science (Tokyo, Japan)] at $40^{\circ} \mathrm{C}$. The eluate was analyzed with a fluorescence detector set to an excitation wavelength and emission wavelength of $380 \mathrm{~nm}$ and $510 \mathrm{~nm}$, respectively. The separation of the samples was performed in succession every $7 \mathrm{~min}$.

Determination of cysteine by HPLC using NAM HPLC conditions and NAM derivative conditions were chosen by referring to previous reports (Nara et al., 1978; Takahashi et al., 1979; Meguro et al., 1996) and the reagent manufacturer's instruction, under conditions as follows: samples were dissolved in $0.25 \mathrm{~mol} / \mathrm{L}$ sodium acetate buffer containing $10 \mathrm{mmol} / \mathrm{L}$ EDTA $(\mathrm{pH} 4.0)$ at predetermined concentrations. NAM was dissolved in acetone to prepare a $1 \mathrm{mmol} / \mathrm{L}$ solution. Then, the cysteine standard solution or the sample solution was mixed with the same volume of NAM solution and incubated for $20 \mathrm{~min}$ at $40^{\circ} \mathrm{C}$. Within $5 \mathrm{~min}$, the reactant was filtered through a $0.45 \mu \mathrm{m}$ filter and $10 \mu \mathrm{L}$ of the filtered reactant was injected into the mobile phase consisting of 10 mmol/L sodium acetate buffer ( $\mathrm{pH}$ 6.0)/methanol (63:37). The flow rate of the mobile phase was $1.0 \mathrm{~mL} / \mathrm{min}$, and the separation was performed with an Inertsil ODS-3 column (5 $\mu \mathrm{m} 4.6 \mathrm{~mm} \phi \times 250 \mathrm{~mm}$; GL science) at $40^{\circ} \mathrm{C}$. The eluate was analyzed with a fluorescence detector set to the excitation wavelength and the emission wavelength of $360 \mathrm{~nm}$ and $420 \mathrm{~nm}$, respectively. The main peak with a retention time of 13.34 min was used for quantification. The separation of the samples was performed in succession every $40 \mathrm{~min}$.

Calculation of cysteine content in powder form of food materials The cysteine content was calculated based on the dry matter. The dry matter was estimated as follows: food materials in powder form were heated at $104^{\circ} \mathrm{C}$ for $3 \mathrm{~h}$ to remove the attached water. The weight was measured and the dry matter calculated after cooling the sample.

Recovery study of ABD-F method and NAM method for Yeast Extract Brand $A$ and Brand $B \quad$ Cysteine was added into each yeast extract so that the amount of addition gave the half, the same, or the double of its cysteine amount. Then each sample was dissolved and the recovery of cysteine was evaluated. Yeast Extract Brand A sample was dissolved at $3 \mathrm{mg} / \mathrm{mL}$. Yeast Extract Brand B sample was dissolved at 3 $\mathrm{mg} / \mathrm{mL}$ for ABD-F method and dissolved at $300 \mu \mathrm{g} / \mathrm{mL}$ for NAM method.

Statistical analysis Data were presented as the mean. Statistical analysis was performed using least squares method. Excel 2003 was used as the statistical analysis software.

\section{Results}

Separation of cysteine derivatives with $A B D-F$ The peak of the cysteine derivative in standard solution was detected at a retention time of approximately $1.82 \mathrm{~min}$. The peak of the cysteine derivative in a model sample solution also was detected at a retention time of approximately $1.82 \mathrm{~min}$; small peaks were detected after $1.82 \mathrm{~min}$, but no peak was detected after $7 \mathrm{~min}$ as shown in Fig. 1. Thus, adequate resolution was obtained within 7 min using this HPLC method.

Calibration curve of cysteine derivative with $A B D-F$ Figure 2 shows the calibration curve of cysteine. Cysteine responded linearly at $r=0.999$ over the range of $0.4 \mathrm{nmol} / \mathrm{L}$ 


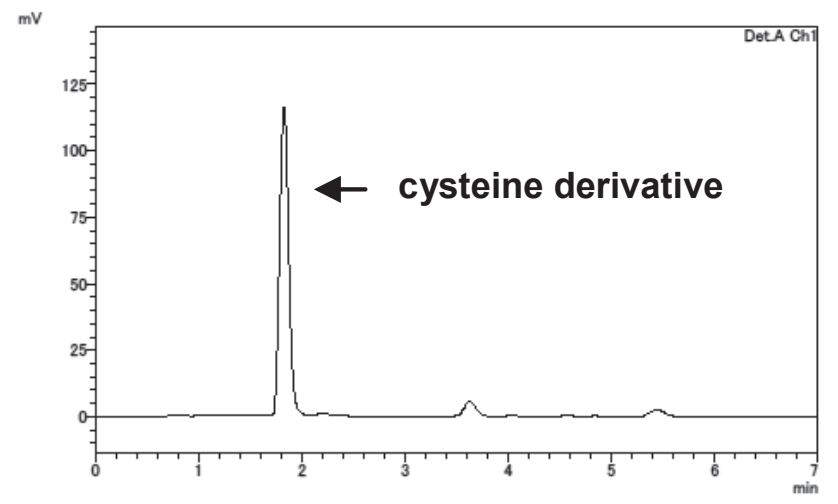

Fig. 1. Chromatogram of the separation of cysteine derivative with $\mathrm{ABD}-\mathrm{F}$ in a model powder sample containing $1 \%$ cysteine on a dry matter basis.

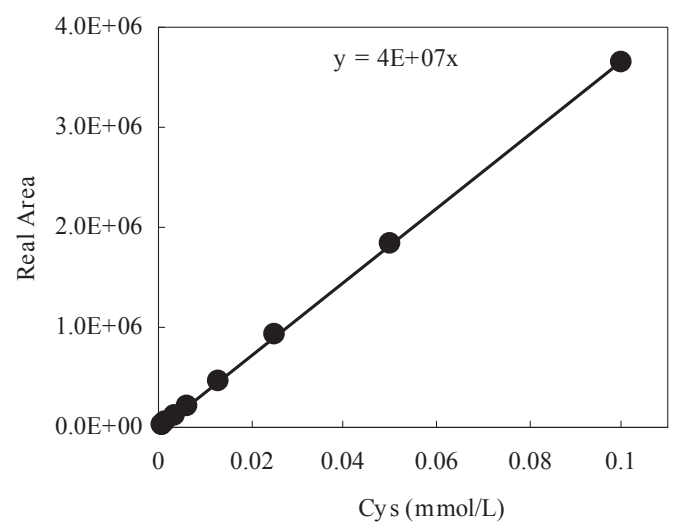

Fig. 2. Calibration curve of cysteine. Cysteine solutions at concentrations from $0.4 \mathrm{nmol} / \mathrm{L}$ to $0.1 \mathrm{mmol} / \mathrm{L}$ were derivatized by ABD-F. Observed peak area was plotted against cysteine solution concentration.

to $0.1 \mathrm{mmol} / \mathrm{L}$. The detection limit was $0.2 \mathrm{nmol} / \mathrm{L}$ when the signal-to-noise ratio was 3 .

Recovery study of added cysteine in a model sample

Table 1 shows the specificity of this method in recovery assays in model sample solutions with cysteine of various concentrations. Added cysteine responded linearly at $r=0.999$ over the range of $0.01 \mathrm{mmol} / \mathrm{L}$ to $0.1 \mathrm{mmol} / \mathrm{L}$ under the test conditions.

Precision study of cysteine derivative with $A B D-F \quad$ To determine the precision of this method, the cysteine derivative in model samples was repeatedly analyzed $(n=25)$. The coefficients of variation $(\mathrm{CV})$ of cysteine were about $1.0 \%$ for the model sample solution containing $0.01 \mathrm{mmol} / \mathrm{L}$ cysteine, about $0.8 \%$ for the $0.05 \mathrm{mmol} / \mathrm{L}$ solution, and about $0.9 \%$ for the $0.1 \mathrm{mmol} / \mathrm{L}$ solution.

Stability of cysteine derivatives with ABD-F during storage The stability of the cysteine derivative was evaluated. Figure 3 shows the relative peak area of the cysteine derivative with ABD-F. Both the relative peak areas for the standard solution and the model liquid food samples were stable
Table 1. Recovery study of cysteine added into model liquid samples.

\begin{tabular}{cccc}
\hline $\begin{array}{c}\text { Amount of added } \\
\text { as final concentration } \\
(\mathrm{mmol} / \mathrm{L})\end{array}$ & $\mathrm{n}$ & $\begin{array}{c}\text { Average } \\
\text { recovery } \\
(\mathrm{mmol} / \mathrm{L})\end{array}$ & $\begin{array}{c}\mathrm{CV} \text { of } \\
\text { recovery } \\
(\%)\end{array}$ \\
\hline 0.010 & 5 & 0.009 & 2.0 \\
0.025 & 5 & 0.024 & 1.2 \\
0.050 & 5 & 0.050 & 1.4 \\
0.100 & 5 & 0.101 & 1.0 \\
\hline
\end{tabular}

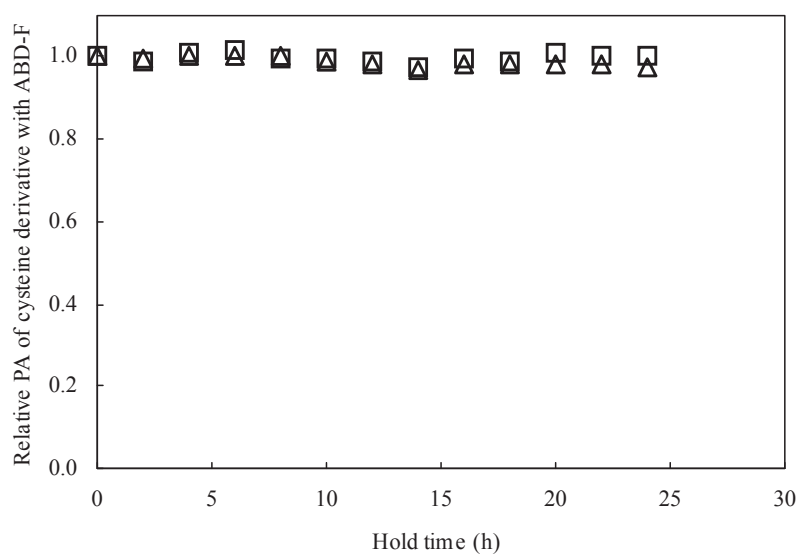

Fig. 3. Stability analysis of the cysteine derivative with ABD-F. The change in relative value of the peak area was plotted every two hours during storage at $4^{\circ} \mathrm{C}$. ( $\square$ ) indicates derivatives of standard cysteine; $(\triangle)$ indicates derivatives of cysteine in a model liquid sample.

and gave a similar value during storage for $24 \mathrm{~h}$ at $4^{\circ} \mathrm{C}$. The actual $\mathrm{CV}$ was less than $2.0 \%$ during this period.

The effect of solvent to dissolve powder form of model samples with ABD-F method Figure 4(a) shows the effect of EDTA concentration in the buffer on the analysis of the model sample powder. Increasing the EDTA concentration in the buffer, increased the measured cysteine content in the model sample powders, which reached a maximum value when EDTA concentration was greater than $1.5 \mathrm{mmol} / \mathrm{L}$. However, this value was about $90 \%$ of the theoretical value calculated from the amount of the cysteine added. Then the effect of the concentration of sodium acetate buffer ( $\mathrm{pH} 4.0)$ was evaluated as shown in Fig. 4(b). The measured cysteine content in powder form of the model samples reached a maximum value when $5 \mathrm{mmol} / \mathrm{L}$ sodium acetate buffer $(\mathrm{pH}$ 4.0) was employed as the solvent. Within the range of 2.5-25 $\mathrm{mmol} / \mathrm{L}$, the value was almost identical with the theoretical value calculated from the amount of the added cysteine. Thus $5 \mathrm{mmol} / \mathrm{L}$ sodium acetate buffer ( $\mathrm{pH} 4.0$ ) containing 1.5 
(a)
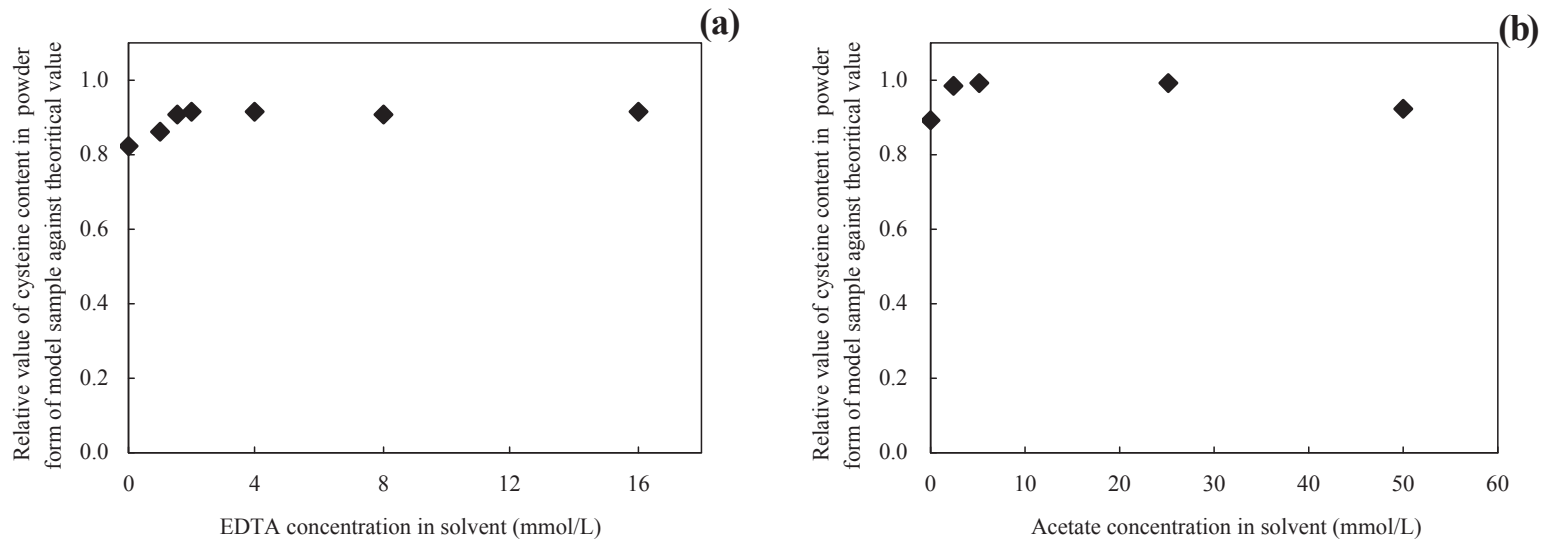

Fig. 4. Effects of buffer components used to dissolve model sample powder containing $1.25 \%$ cysteine on the dry matter basis: (a) Effect of EDTA concentration; (b) Effect of sodium acetate buffer concentration ( $\mathrm{pH} 4.0$ ).
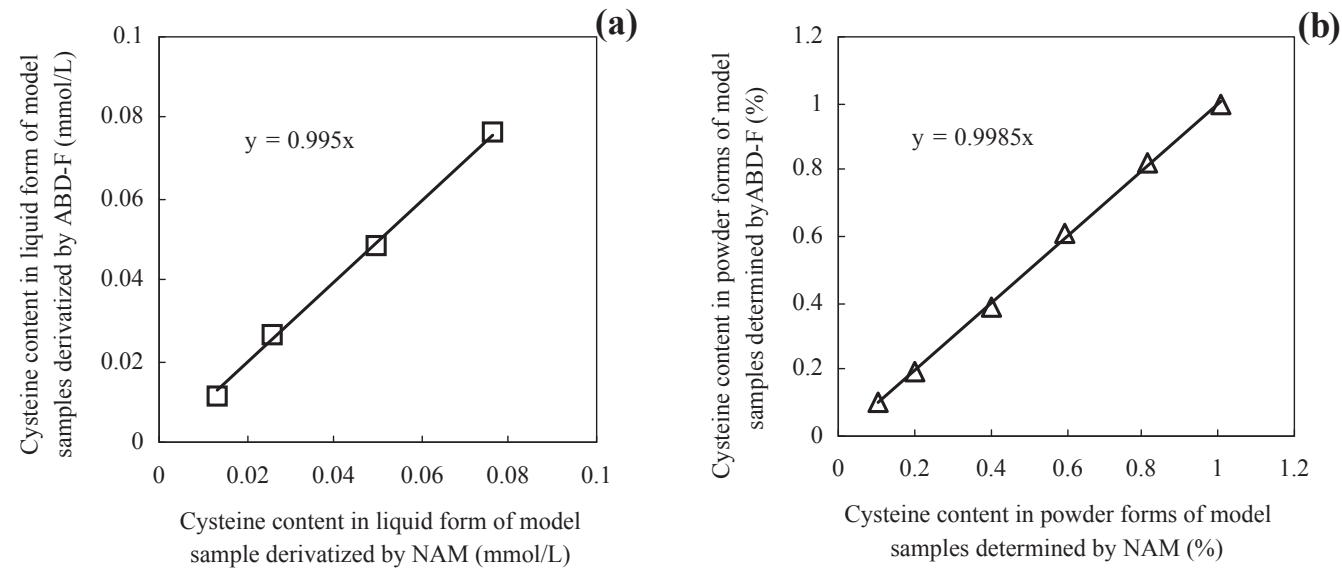

Fig. 5. Comparison of cysteine content in samples determined by ABD-F and by NAM: (a) Evaluation of the method used for liquid samples; (b) Evaluation of the method used for powder samples.

$\mathrm{mmol} / \mathrm{L}$ EDTA was used as a solvent for further experiments.

Accuracy of cysteine content determined by the ABD-F method compared to the NAM method The cysteine content in model samples was determined by both the ABD-F and the NAM methods. As shown in Fig. 5(a), the cysteine content in liquid samples as determined by the ABD-F method was similar to that as determined by the NAM method, with $\mathrm{r}=0.999$ over the range from $0.01 \mathrm{mmol} / \mathrm{L}$ to $0.08 \mathrm{mmol} / \mathrm{L}$. As shown in Fig. 5(b), cysteine content in powder samples as determined by the ABD-F method was similar to the content as determined by the NAM method, with $r=0.999$ over the range from $0.1 \%$ to $1.0 \%$ on a dry matter basis.

\section{Determination of cysteine content of food materials}

The cysteine content of six types of food materials was determined using the ABD-F method and the NAM method. As shown in Table 2, the cysteine content as determined by ABD-F was almost identical to that as determined by NAM for four types of food materials. But the values determined using ABD-F were smaller than those from NAM for two types of food materials.
Recovery study of added cysteine from two types of food materials in powder form As shown in Table 3, good recovery of added cysteine was found for both the ABD-F and NAM methods for yeast extract Brand A. For yeast extract Brand $\mathrm{B}$, good recovery of added cysteine was found for the ABD-F method but not for the NAM method.

\section{Discussion}

Specificity, linearity, and precision were verified for the ABD-F method to determine cysteine content in model samples. As shown in Fig. 5, the cysteine content as determined using the ABD-F method was similar to that as determined by NAM, which indicates that the ABD-F method can be considered as accurate as the NAM method for cysteine in amounts greater than $0.1 \%$ on a dry matter basis.

In this method, sodium acetate buffer containing $2 \mathrm{mmol} /$ L EDTA ( $\mathrm{pH} 4.0$ ) was employed to dilute food materials. When water or other buffer with a near neutral $\mathrm{pH}$ was used as a solvent to dissolve food materials in powder form, reproducible results were not obtained in some cases, and, 
in the worst cases, $\mathrm{CV}$ was approximately $10 \%(\mathrm{n}=5)$. According to a study of oxidation of thiol compounds, selfoxidation depends linearly on the concentration of dissolved oxygen (Cavallini et al., 1968). In addition, the oxidation rate in neutral and weak alkaline buffer increases 100-fold in the presense of catalytic amounts of metal ions, although thiol coumpounds are resistant to dissolved oxygen in acidic

Table 2. Cysteine content of six types of food materials as determined by the ABD-F and NAM methods.

\begin{tabular}{lccc}
\hline & & \multicolumn{2}{c}{ Contents of Cys in food materials $(\mathrm{g} / 100 \mathrm{~g})$} \\
\hline Yeast Extract & ABD-F & NAM \\
& Brand A & 0.027 & 0.028 \\
& Brand B & 0.060 & 0.108 \\
& Brand C & 0.015 & 0.014 \\
Chicken Extract & Brand D & 0.082 & 0.124 \\
Pork Extract & & 0.032 & 0.034 \\
\hline
\end{tabular}

buffer (Bagiyan et al., 2003). Some food materials in powder form are very sticky and tend to form a lump during dilution. In such cases, samples need to be shaken vigorously with diluting solvent, which increases dissolved oxygen concentraion in the solution. As a result, cysteine in the food materials can be oxidized by the metal ions in the food. However, sodium acetate buffer ( $\mathrm{pH} 4.0$ ) containing 2 mmol/L EDTA may prevent such oxidation if the appropriate concentration of the buffer is used and does not inhibit the ABD-F derivation.

Using this ABD-F method, the cysteine content of six types of food materials in powder form was measured and compared with those obtained using the NAM method. As shown in Table 2, the cysteine content of four types of food materials as determined by ABD-F was identical to that determined by NAM, but the cysteine content of two types of food materials was different than that determined by NAM.

Table 3. Recovery study of the ABD-F and NAM methods for Yeast Extract Brands A and B.

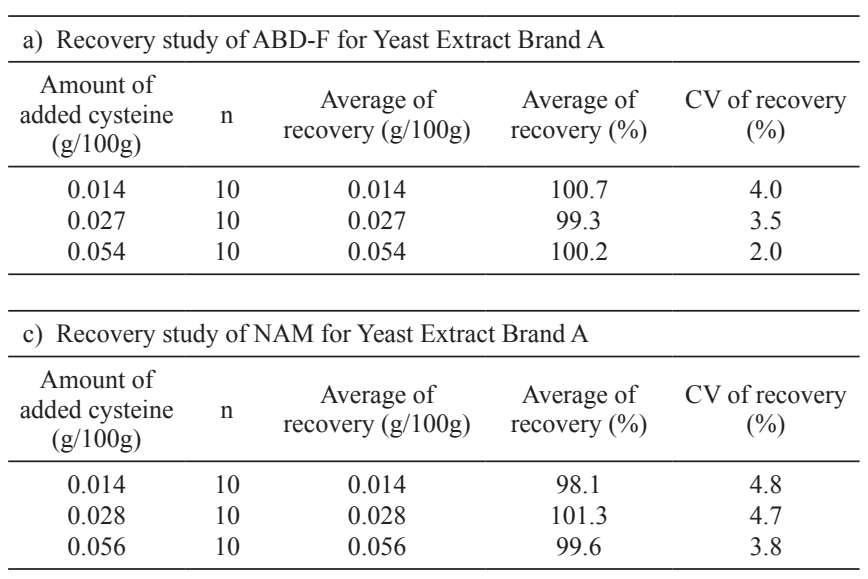

\begin{tabular}{|c|c|c|c|c|}
\hline \multicolumn{5}{|c|}{ b) Recovery study of ABD-F for Yeast Extract Brand B } \\
\hline $\begin{array}{l}\text { Amount of } \\
\text { added cysteine } \\
(\mathrm{g} / 100 \mathrm{~g})\end{array}$ & $\mathrm{n}$ & $\begin{array}{c}\text { Average of } \\
\text { recovery }(\mathrm{g} / 100 \mathrm{~g})\end{array}$ & $\begin{array}{l}\text { Average of } \\
\text { recovery }(\%)\end{array}$ & $\begin{array}{c}\text { CV of recovery } \\
(\%)\end{array}$ \\
\hline 0.030 & 10 & 0.031 & 101.0 & 4.1 \\
\hline 0.060 & 10 & 0.061 & 100.8 & 2.7 \\
\hline 0.120 & 10 & 0.120 & 99.8 & 1.8 \\
\hline \multicolumn{5}{|c|}{ d) Recovery study of NAM for Yeast Extract Brand B } \\
\hline $\begin{array}{l}\text { Amount of } \\
\text { added cysteine } \\
(\mathrm{g} / 100 \mathrm{~g})\end{array}$ & $\mathrm{n}$ & $\begin{array}{c}\text { Average of } \\
\text { recovery }(\mathrm{g} / 100 \mathrm{~g})\end{array}$ & $\begin{array}{l}\text { Average of } \\
\text { recovery }(\%)\end{array}$ & $\begin{array}{c}\mathrm{CV} \text { of recovery } \\
(\%)\end{array}$ \\
\hline 0.054 & 10 & 0.043 & 79.1 & 9.2 \\
\hline 0.108 & 10 & 0.095 & 88.2 & 5.7 \\
\hline 0.216 & 10 & 0.244 & 112.9 & 8.1 \\
\hline
\end{tabular}
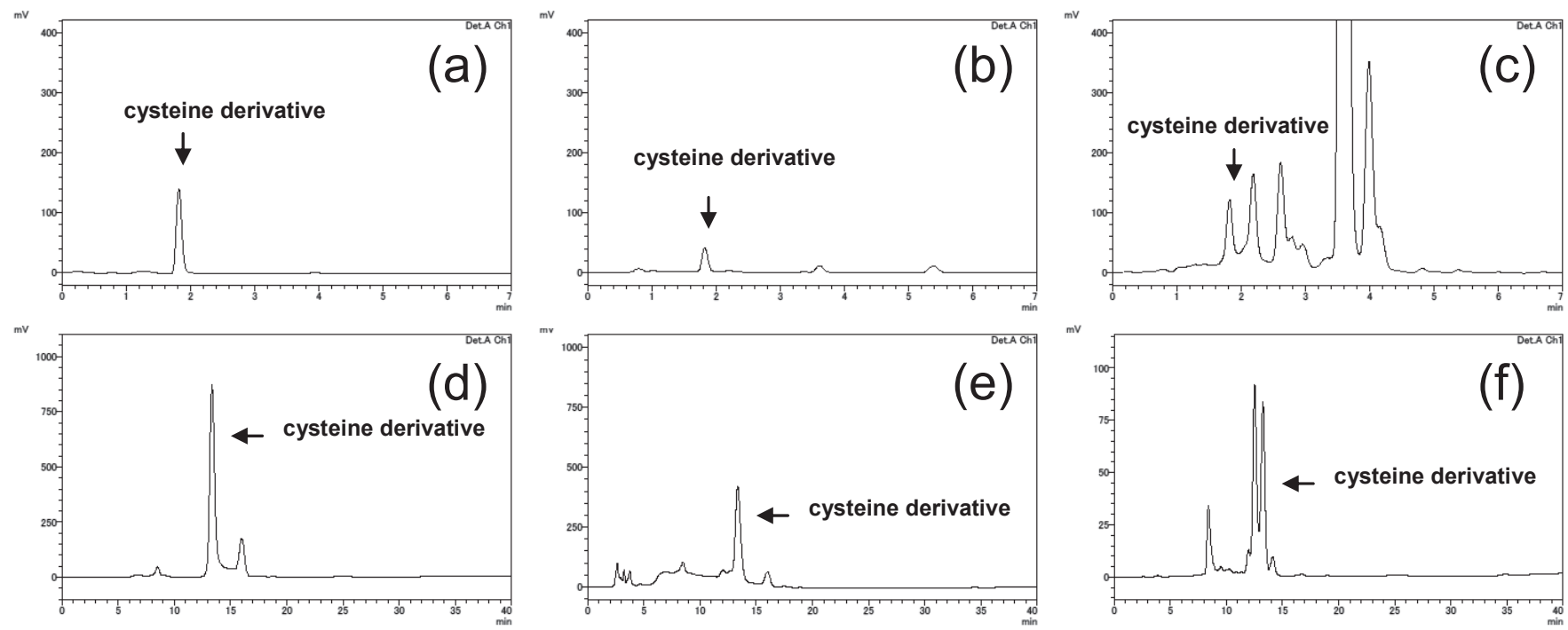

Fig. 6. Chromatogram of cysteine derivatives with ABD-F or NAM: (a)-(c) Derivatives with ABD-F; (d) -(f) Derivatives with NAM. 6(a) and (d) Cysteine standard (2 mg/L); (b) and (e) Yeast extract brand A (3 mg/mL); (c) Yeast extract brand B (3 mg/mL); (f) Yeast extract brand $\mathrm{B}(300 \mu \mathrm{g} / \mathrm{mL})$. 
Therefore, one yeast extract (brand A) was used as a representative of the former and another (brand B) was used as a representative of the latter to analyze the reason for these differences.

As shown in Fig. 6, the cysteine derivatives with ABD-F and with NAM were detected as a main peak for brand A. For brand $\mathrm{B}$, the cysteine derivative with ABD-F was detected as a separate peak; however, the cysteine derivative with NAM was not separated. This might be due to the nature of NAM. As shown in Figs. 6(a) and (d), the cysteine derivative was detected as a single peak with ABD-F, but the derivative with NAM was detected in three peaks; the biggest peak was detected at a retention time of approximately $13.34 \mathrm{~min}$, the second peak at a retention time of approximately $15.99 \mathrm{~min}$, and a third peak at a retention time of approximately 8.49 min. The biggest peak was used for quantification since the proportion of these three peaks was the same even when the concentration of the cysteine standard solution was varied. In addition, the cysteine content in the model samples was the same when only the biggest peak was used for quantification or when the sum of the three peaks was used for quantification. However, some of the food materials, such as yeast extract brand $\mathrm{B}$, might contain much sulfur compounds other than cysteine. The derivatives of such compounds could give several peaks and prevent sufficient separation of the cysteine derivative. When the glutathione derivative with NAM was quantified using a pre-column method the glutathione derivative with NAM also was detected with several peaks (Hashimoto et al., 1992). Several peaks might be produced from the nature of NAM. Thus, ABD-F was more suitable for analyzing the cysteine content of food materials because it gave a single peak for cysteine.

These results indicate that this ABD-F method allows rapid and precise determination of cysteine content in food materials and can be useful for the food industry.

Acknowledgements We sincerely thank Dr. Mitsuaki Mori at Japan Food Research Center for his technical advice on determination of cysteine by HPLC using NAM. We also sincerely thank Dr. Motonaka Kuroda at Ajinomoto for his advice and evaluation.

\section{References}

Bagiyan, G.A., Koroleva, I.K., Soroka, N.V. and Ufimtsev, A.V. (2003). Oxidation of thiol compounds by molecular oxygen in aqueous solutions. Russian Chemical Bulletin, International Edition., 52 (5), 1135-1141.

Cavallini, D., De Marko, C. and Dupre, S. (1968) Luminol Chemiluminescence Studies of the Oxidation of Cysteine and Other Thiols to Disulfides. Arch. Biochem. Biophys., 124, 18-26.
Grandvoinnet, P. and Berger, M. (1979). Effects of a reducing agent and an oxidant on wheat flour-based bakery products. Annales de Technologie Agricole, 28 (3), 287-297.

Hashimoto, M., Iwatsuki, S., Kuwata, G. and Imai, M. (1992) HPLC Determination of Glutathione in Food. Nihon Eiyoushokuryou Gakukaishi, 45 (4), 363-365

Kuroda, M., Nagaba, H., Tsubuku, T., Kawajiri, H. and Ueda, Y. (1997). Simultaneous determination of glutathione, $\gamma$-glutamylcysteine and cysteine in commercial yeast extract by HPLC with fluorometric detection. Food Sci. Technol. Res., 3 (3), 239-240.

Meguro, H. and Ohrui, H. (1996).Strategy and design of novel reagents for the fluorometric analysis of biomolecules. Biosci. Biotech. Biochem., 60 (12), 1919-1924.

Naim, M., Wainish, S., Zehavi, U., Peleg, H., Rouseff, R.L. and Nagy, S. (1993) Inhibition by thiol compounds of off-flavor formation in stored orange juice. 1. Effect of L-cysteine and Nacetyl-L-cysteine on 2,5-dimethyl-4-hydroxy-3(2H)-franone formation. J. Agric. Food. Chem., 41, 1355-1358.

Naim, M., Zuker, I., Zehavi, U. and Nagy, S. (1993) Inhibition by thiol compounds of off-flavor formation in stored orange juice. 2. Effect of L-cysteine and N-acetyl-L-cysteine on p-vinylguaniacol formation. J. Agric. Food. Chem., 41, 1355-1358.

Nara, Y. and Tuzimura, K. (1978). Synthesis of N-(9-Acridinyl)maleimide, a fluorometric reagent for thiol compounds. Agric. Biol. Chem., 42 (4), 793-798.

Reddy, M.N. and Behnke, C. (1997). A rapid and simple assay to homocysteine and other thiols in pediatric samples by high pressure liquid chromatography and fluorescence detection. J. Liq. Chrom. \& Rel. Technol., 20 (9), 1391-1408.

Starkenmann, C., Troccaz, M. and Howell, K. (2008). The role of cysteine and cysteine-S conjugates as odour precursors in the flavour and fragrance industry. Flavour Fragra. J., 23, 369-381.

Takahashi, H., Nara, Y., Meguro, H. and Tuzimura, K. (1979). A sensitive fluorometric method for the determination of glutathione and some thiols in blood and mammalian tissues by high performance liquid chromatography. Agric. Biol. Chem., 43 (7), 1439-1445

Toyo'oka, T. and Imai, K. (1984). New fluorogenic reagent having halogenobenzofurazan structure for thiols: 4-(aminosulfonyl)7-fluoro-2,1,3-benzoxadiazole. Analytical Chemistry, 56 (13), 2461-2464.

Treuheit, M.J. and Kirley, T.L. (1993). Reversibility of cysteine labeling by 4-(aminosulfonyl)-7-fluoro-2,1,3-benzoxadiazole. Analytical Biochemistry, 212, 138-142.

Wada, M. and Takagi, H. (2006). Metabolic pathways and biotechnological production of L-cysteine. Appl. Microbiol. Biotechnol., 73, 48-54. 